\begin{tabular}{|c|l|}
\hline Title & Topology optimization of IPM motor with aid of deep learning \\
\hline Author(s) & Sasaki, Hidenori; Igarashi, Hajime \\
\hline Citation & $\begin{array}{l}\text { International journal of applied electromagnetics and mechanics, 59(1), 87-96 } \\
\text { https://doi.org/L0.3233/AA E-171164 }\end{array}$ \\
\hline Issue Date & 2019-03-21 \\
\hline Doc URL & http://hdl.handle.net/2115/73870 \\
\hline Rights & The final publication is available at IOS Press through http://dx.doi.org/10.3233/AAE-171219 \\
\hline Type & article (author version) \\
\hline File Information & ISEM2017.pdf \\
\hline
\end{tabular}

Instructions for use 


\title{
Topology Optimization of IPM Motor with Aid of Deep Learning
}

\author{
Hidenori SASAKI ${ }^{a, * *}$ and Hajime IGARASHI ${ }^{a}$ \\ ${ }^{a}$ Graduate School of Information Science and Technology, Hokkaido University, 060-0814, Japan
}

\begin{abstract}
This paper presents a new topology optimization of interior permanent magnet (IPM) motors using the genetic algorithm with aid of the deep leaning. The data composed of the rotor shape of an IPM motor and its performance, obtained by a prior topology optimization process, is input to a convolutional neural network (CNN). After the learning process, CNN is shown to provide fairly accurate estimate of the motor performance. During the posterior topology optimization, the finite element analysis (FEA) is carried out only for the limited number of individuals; probability that FEA is performed increases with the motor performance evaluated by CNN. It is shown that the computing time is reduced to about $1 / 10$ without deterioration of the optimization performance with aid of the deep learning.
\end{abstract}

Keywords: Topology Shape Optimization, Deep learning, Finite Element Method, IPM motor

\section{Introduction}

Recently, development of high-performance motors has become considerably important for realization of high-efficiency electric vehicles. In development of such motors, it is necessary to consider various the motor characteristics as well as constraints relevant to torque, demagnetizations, mechanical vibrations, mechanical strength and so on in a limited period of time. For this reason, it has become difficult to meet all the requirements when we rely only on knowledge and experience of the designer. The optimization method using electromagnetic field analysis with the finite element method (FEM) is fairly effective to overcome this difficulty.

We consider the topology optimization for the design of interior permanent magnet (IPM) motors in this study. The parameter optimization, widely used in industries, searches for the optimal values of, e.g., length, location and angle. On the other hand, the topology optimization freely modifies the shape including generation and annihilation of holes. The latter method is expected to lead to innovative motor shapes. The topology optimization based on the genetic algorithm (GA) in which the core shape is represented by the Gaussian basis functions has been shown to work quite well for design of rotating machines [1, 2]. It is, however, computationally expensive to perform such topology optimization because a number of fitness evaluations with FEM are involved.

For the parameter optimization, time-efficient surrogate methods such as the response surface method [3] and Kriging method [4] have been proposed. In these methods, the surrogate model is constructed on the basis of the FE analysis at sampling points prior to the optimization. During the optimization, the fitness is evaluated using the surrogate model. Although these methods work well for optimization problems with relatively small degrees of freedom (DoFs), they would be unsuitable for the topology optimization which has a few tens of DoFs and more. That is, if we set $n$ sampling points for each DoF in the conventional surrogate model, the total number of sampling points increases in proportion to $n^{\text {DoFs }}$. The surrogate model becomes computationally prohibitive when DoFs are large as in the topology optimization.

In this study, as a new surrogate method, we employ the deep learning which has had great successes in image recognition, speech recognition, and so on [5]-[7]. Unlike the conventional surrogate methods, the deep learning can directly treat with images and automatically extract the local features from them for classification. Hence there is no difficulties which are mentioned above in the deep learning. In the proposed method, the data composed of the rotor shape of an IPM motor and its performance, obtained by a prior topology optimization process, is input to a convolutional neural network (CNN) $[8,9]$. During the posterior topology optimization, the finite element analysis (FEA) is carried out only for the limited number of individuals; probability that FEA is performed increases with the motor performance evaluated by CNN. The performance of this method will be evaluated through numerical experiments. Finally, the potential use of the proposed method for optimal design will be discussed. 


\subsection{NGnet-on/off method}

For the topology optimization, we use the NGnet- on/off method based on GA [1, 2]. This method has successfully been applied to the topology optimization of the rotor of IPM motors to improve the torque characteristics. As shown Fig. 1, the design region of an IPM motor is subdivided into finite elements. The material attribute $V_{\mathrm{e}}$, of element e, iron or air, in the design region is determined in such a way that $V_{e}=$ iron (air) if $\phi \geq 0(\phi<0)$ where $\phi$ is the shape function defined by

$$
\begin{aligned}
& \phi(x)=\sum_{i=1}^{N} w_{i} b_{i}(\boldsymbol{x}) \\
& b_{i}(\boldsymbol{x})=G_{i}(\boldsymbol{x}) / \sum_{k=1}^{N} G_{k}(\boldsymbol{x})
\end{aligned}
$$

where, $G$ and $\boldsymbol{x}$ denote the Gaussian function and position vector, respectively. The Gaussians are uniformly distributed in the design region. The iron distribution in the design region would change in response to the change in the weighting coefficients $\boldsymbol{w}=\left(w_{1}, w_{2}, \ldots, w_{N}\right)^{t}$. We use the real-coded genetic algorithm (RGA) for determination of $\boldsymbol{w}$ so that the torque performance $F$ of the IPM motor shown in Fig. 1 is maximized.

\subsection{Problems in conventional optimization based on GA}

In the initial phase of RGA, many individuals with different rotor shapes are generated randomly. The typical distribution of the torque performance (fitness) of these initial individuals is shown in Fig. 2. The fitness of 73.5\% individuals, shown in red, is lower than half of the original fitness of the IPM motor before the optimization. These individuals are unlikely to survive to the next generation. In the next generations, this tendency would remain; most of the individuals have poor fitness and only a part of individuals has the fitness comparable to that of the elite individual. From this tendency, which is common for population-based optimization algorithms, we come up with an idea: the fitness of all the individuals do not has to be evaluated by the time consuming FEM. Instead, the fitness is evaluated by a time-efficient surrogate method, and only the individuals to which the surrogate method gives good score are evaluated by FEM. For the surrogate method, we employ here the deep learning based on CNN.

\subsection{Convolutional Neural Network}

For the deep learning, we use GoogLenet [10] based on CNN, which won ILSVRC - 2014, schematically shown in Fig. 3. As shown in Fig.3, CNN consists of the convolution, pooling and output layers, and has 22 layers in total. The convolution layer performs filter operation in which the input data are multiplied by the weights and summed up. When appropriate values are set to the weights, a local feature of the input image can be extracted. The local units in the convolution layer form a receptive field. The outputs of a receptive field are input to the pooling layer, where small inputs are filtered out to make CNN robust. The output layer outputs the classification probability $p$ computed from the Soft Max function

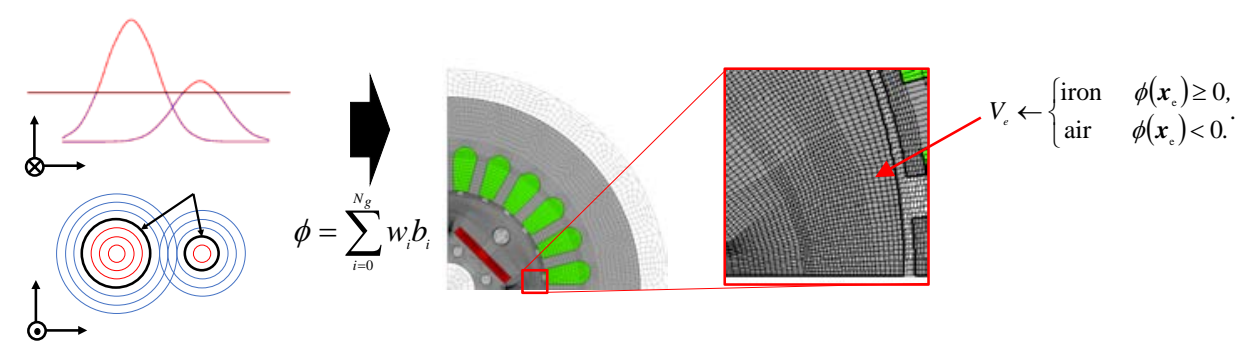

Fig. 1. NGnet-on/off method.

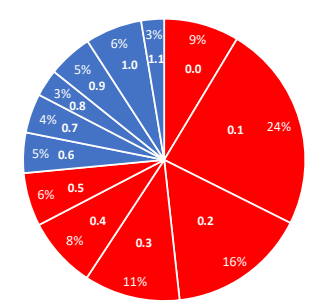

Fig. 2. Distribution of fitness in the initial population 


$$
p_{j}=\frac{e^{x_{j}}}{\sum_{k=1}^{n} e^{x_{k}}},
$$

where, $e, x$ and $n$ are Napier's constant, the input to the output layer and the number of input, respectively. The composition of the GoogLenet is based on CNN. In this study, we use Caffe library to configure CNN [12].

The image classification can also be performed by other machine learning methods such as Bayesian method, support vector machines and so on. The one of the demerits of these methods is that we need to set the appropriate features for image classification. However, during the topology optimization process, we generate diverse motor shapes, for all of which it is difficult to set the appropriate features by hand. On the contrary, the deep learning automatically extracts the local image features from the input image for classification. The deep leaning is, therefore, very suitable for the topology optimization.

\subsection{Topology Optimization with Aid of Deep Learning}

In the phase prior to the optimization, the relations between the rotor shape of an IPM motor, characterized by the weighting coefficient $\boldsymbol{w}$, and its performance are computed through test topology optimization processes. These relations are used as the training data input to a convolutional neural network (CNN). The resultant CNN is used for the surrogate model for the topology optimizations starting from different random seeds and possibly with different motor specifications. During the topology optimization posterior to this initial leaning phase, the individuals are classified under the performance by CNN. Then, the fitness of the limited number of individuals is re-evaluated by FEM at high accuracy. The probability for the FEM evaluation dependent on the performance evaluated by CNN is set as shown in Fig.4. Note that the probability becomes higher with the expected performance. Because we can reduce the number of FE computations by this method, the computational time is expected to be shortened. When we excessively skip the FE computations, however, the performance of the optimized IPM motor would be deteriorated. This point will be discussed in the next section.

\section{Numerical Results}

\subsection{Performance of Deep Learning}

Figs. 5 (a) and (b) show the original model of an IPM motor before optimization and the supports of the Gaussians, with a certain threshold, represented by the circles, which are uniformly distributed in the design region [11]. The average torque of the original model is $2.08 \mathrm{Nm}$. In this study, the average torque of the IPM motor is maximized. The optimization problem is defined by

$$
F=\frac{T}{T^{0}} \rightarrow \text { max., Sub.to. } N_{\text {area }}<2,
$$

where, $T$ and $T_{0}$ denote the average torque and that of the original model, and $N_{\text {area }}$ is the number of separated rotor cores. This constraint imposes that the rotor core is not separated into parts. Table. 1 shows the optimization and motor settings.

We perform the topology optimization of the IPM motor once to generate the training data for CNN. During this optimization, we perform $800+160 \times 100$ fitness evaluations. There are many individuals with low fitness and, on the contrary, small number of ones with high fitness. For the training of CNN, however, the fitness distribution should be as uniform as possible. For this reason, we select 4,000 from 16,800 data to have the nearly uniform training data. The spatial distribution of the materials such as magnet, iron core, air corresponding to flux barriers in the rotor, is expressed in a bitmap image and

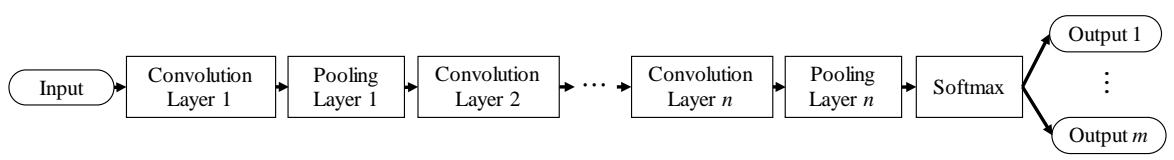

Fig. 3. Convolution Neural Networks [8]

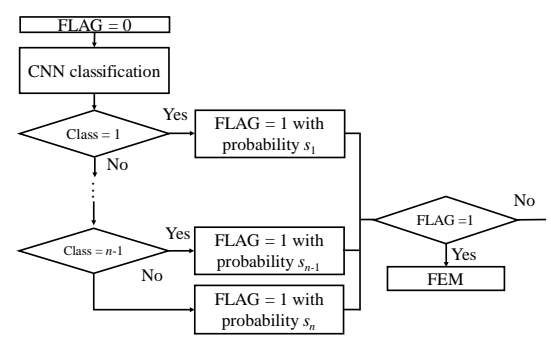

Fig. 4. Flow diagram of fitness evaluation 
input to CNN, which is supervised to output the corresponding fitness. The individuals are classified into 8 categories by CNN with respect to the fitness computed by FEM as shown Table. 2.

In order to test the performance of $\mathrm{CNN}$, we evaluate the fitness values of other 4000 individuals, and compare them with the correct values computed by FEM. Table. 3 summarizes the relationship between evaluation fitness evaluated by CNN. The columns are the results of CNN while the rows are the results of FEM. The individuals in the diagonal of this table are classified by CNN without errors. The individuals near the diagonal are classified by CNN with small error. The error distribution of the classification is summarized in Table. 4, from which we see that the classification error $e$ is less than 0.1 for over $99 \%$. In conclusion, CNN has fairly good accuracy for its classification of the individuals.

Figs. 6 (a)-(d) show the bitmap images which are input to CNN. The regions colored gray, red, green and white correspond to iron, permanent magnetic, copper and air, respectively. The pair of numbers under the bitmaps represent the fitness computed by CNN and FEM. They are found to be in good agreement. The motor shown in Fig. 6 (a) has poor torque performance because of the small flux

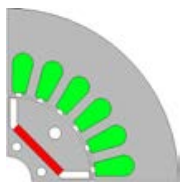

(a) Original model

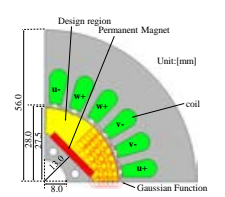

(b) Design region

Fig. 5. IPM motor

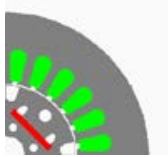

(a) $(0.5,0.5)$

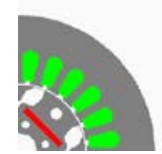

(b) $(0.7,0.7)$

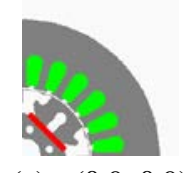

(c) $(0.9,0.9)$

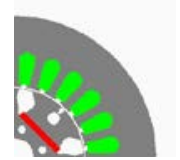

(d) $(1.1,1.1)$

Fig. 6. Bitmaps ( $\left.F_{\mathrm{CNN}}, F_{\mathrm{FEM}}\right)$

Table. 1.

Optimization and motor setting.

\begin{tabular}{|c|c|}
\hline Number of genes & 42 \\
\hline Number of individuals & 800 \\
\hline Number of children & 160 \\
\hline Number of generations & 100 \\
\hline Current phase angle [degree] & 30 \\
\hline Current effective value [A] & 4.2425 \\
\hline Number of turns [turn] & 35 \\
\hline Residual flux densitv [T] & 1.25 \\
\hline Number of genes & 42 \\
\hline
\end{tabular}

Table. 2.

Classification of IPM motors

\begin{tabular}{|c|c|}
\hline CNN Output & FEM Output \\
\hline 0 & $\sim 0.45$ \\
\hline 0.5 & $0.45 \sim 0.55$ \\
\hline 0.6 & $0.55 \sim 0.65$ \\
\hline 0.7 & $0.65 \sim 0.75$ \\
\hline 0.8 & $0.75 \sim 0.85$ \\
\hline 0.9 & $0.85 \sim 0.95$ \\
\hline 1 & $0.95 \sim 1.05$ \\
\hline 1.1 & $1.05 \sim$ \\
\hline
\end{tabular}

Table. 3.

Class labels resulted from FEM and CNN

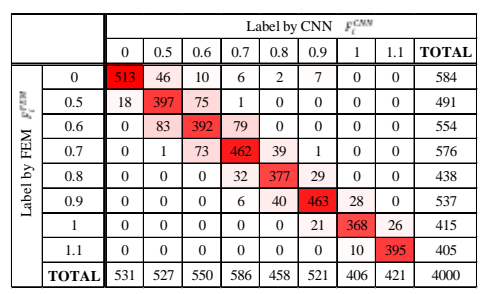


Table. 4.

Error distribution

\begin{tabular}{|c|c|c|}
\hline Classification error $e$ & Number of individuals & Ratio[\%] \\
\hline 0 & 3367 & 84.2 \\
\hline 0.1 & 599 & 15.0 \\
\hline 0.2 & 19 & 0.5 \\
\hline 0.3 & 6 & 0.2 \\
\hline 0.4 & 2 & 0.1 \\
\hline 0.5 & 7 & 0.2 \\
\hline 0.6 & 0 & 0.0 \\
\hline 0.7 & 0 & 0.0 \\
\hline
\end{tabular}

barriers. On the other hand, the motor shown in Fig. 6 (d) has much better torque performance because of the large flux barriers which guide the magnetic flux from the magnet to the stator. We conclude that the classification of CNN is fairly accurate. Note here that CNN estimates the performance of the motor only from the material distribution within computational time much shorter than that of FEM.

In the topology optimization followed by the learning phase of CNN, FE computations for evaluation of the fitness are performed according to the probability distribution indicated in Table. 5. Distribution (i) corresponds to the conventional optimization using only FEM. When distribution (ii) is employed, all the individuals in the best class are evaluated by FEM while the probability of the FEM computations is reduced along with decrease in the fitness evaluated by CNN. When distribution (iii) is employed, the FEM computations are further reduced.

\subsection{Result of Topology Optimization}

Fig. 7 shows the optimized rotor shape of the IPM motor for the probability distributions (i), (ii) and (iii). There are no significant differences in the performance of optimal solutions. The numbers of FEM evaluation are plotted against generations of GA in Fig. 8. All the individuals are evaluated by FEM in case (a). The number of FEM evaluation gradually increases because the number of individuals classified into the upper classes increases along with the generation. When we employ the probability distribution (c), the computing time is reduced to about 1/10 in comparison with the optimization based on (a).

\section{Possible Acceleration of Optimization Using Present Method}

In the above example, CNN is supervised to output the torque performance of the IPM motor. Then CNN is used for the optimization of the same IPM motor. Hence, the total computing time including the learning phase would be longer than the conventional optimization without CNN.

The most promising use of the present method would be for the optimization which consists of the objective function $F$ and constraint function $G$ in the form

$$
\begin{aligned}
& F=\sum_{i=1}^{n} \alpha_{i} F_{i} \\
& G=\sum_{i=1}^{m} \beta_{i} G_{i}
\end{aligned}
$$

where $\alpha, \beta$ are the weighting coefficients. First, we perform an optimization for arbitrary values of the weighting coefficients. After the optimization, $n+m$ CNNs are supervised to output $F_{i}, i=$ $1,2, \ldots, n$ and $G_{i}, i=1,2, \ldots, m$. Then for different weighting coefficients, the optimization can be

Table. 5.

Probabilities of FEM evaluation for different classes

\begin{tabular}{|c|c|c|c|}
\hline \multirow{2}{*}{ CNN Output (class $n$ ) } & \multicolumn{3}{|c|}{ Probability $s_{n}$ for FEM analysis [\%] } \\
\cline { 2 - 4 } & $(\mathrm{I})$ & (II) & (III) \\
\hline $0.0(8)$ & 100 & 1.5625 & 0 \\
\hline $0.5(7)$ & 100 & 3.125 & 1.5625 \\
\hline $0.6(6)$ & 100 & 6.25 & 3.125 \\
\hline $0.7(5)$ & 100 & 12.5 & 6.25 \\
\hline $0.8(4)$ & 100 & 25 & 12.5 \\
\hline $0.9(3)$ & 100 & 50 & 25 \\
\hline $1.0(2)$ & 100 & 100 & 50 \\
\hline $1.1(1)$ & 100 & & \\
\hline
\end{tabular}


accelerated by CNN which has already supervised to output the correct values of $F_{i}, G_{i}$. We often encounter this situation; for example, we first optimize a motor with a large weight to average torque and small one to torque ripple. If we find that torque ripple is too large after the optimization, then we would perform another optimization by increasing the weight to the torque ripple. The second optimization can be accelerated by CNNs supervised for the average torque and torque ripple. Similarly, the present method is expected to accelerate the multi-objective optimization for $\left(F_{1}, F_{2}, \ldots\right)$. These possible accelerations will be verified with numerical examples elsewhere.

\section{Conclusions}

We have proposed a new topology optimization method of IPM motors based on the deep learning. The bitmap image generated from the material distribution of the IPM motor is input to CNN, which is supervised to output the torque performance computed by FEM. The torque performance evaluated by CNN has been shown to agree well with the supervised data. Whether the FEM computations are performed or not is determined with reference to the fitness evaluated by CNN. There are no significant differences in the performance of the optimized IPM motors when we employ the probability distributions of FEM evaluation under test. Using the proposed method, it is possible to reduce the number of FEM evaluations without deterioration in the performance of the topology optimization.

The supervised CNN would be valid even when we make small differences in the magnet shape and position, rotor radius and so on. Moreover, using the data for IPM motors with different design parameters as the input data to CNN, the generalization of CNN would be possible. They are remained for our future works.

\section{References}

[1] T. Sato, K. Watanabe, and H. Igarashi, "Multimaterial Topology Optimization of Electric Machines Based on Normalized Gaussian Network,” IEEE Trans. Magnetics, vol. 51, no. 3, pp. 1-4, 2015.

[2] S. Sato, T. Sato, and H. Igarashi, "Topology optimization of synchronous reluctance motor using normalized Gaussian network,” IEEE Trans. Magnetics, vol.51, no.3, 2015.

[3] J. W. Bandler, et al., "Space mapping technique for electromagnetic optimization,” IEEE Transactions on Microwave Theory and Techniques, vol.42, No. 12, pp. 2536-2544, 1994.

[4] J. B. Kim, K. Y. Hwang, and B. I. Kwon, "Optimization of two-phase in-wheel IPMSM for wide speed range by using the Kriging model based on Latin hypercube sampling,” IEEE Transactions on Magnetics, vol. 47, No. 5, pp. 1078-1081, 2011.

[5] Y. LeCun, B.Yoshua, H. Geoffrey, “Deep learning,” Nature, vol. 521, No. 7553, pp. 436-444, 2015.

[6] D. Silver, et al., "Mastering the game of Go with deep neural networks and tree search,” Nature, vol. 529, No. 7587, pp. 484-489, 2016.

[7] J. Lee, K. Cho, and T. Hofmann, "Fully character-level neural machine translation without explicit segmentation,” arXiv preprint arXiv:1610.03017, 2016.

[8] T.Okatani, "Deep Learning for Image Recognition,” Journal of Japanese Society for Artificial Intelligence, vol.28.6, pp.962-974, 2013. ( in Japanese )

[9] H. Asou, "Deep Representation Learning by Multi-Layer Neural Network," Journal of Japanese Society for Artificial Intelligence, vol. 28, No. 4, pp. 649-659, 2013. ( in Japanese )

[10] C. Szegedy, et al. “Going deeper with convolutions," Proceedings of the IEEE conference on computer vision and pattern recognition, pp. 1-9, 2015.

[11] “Technical report of the institute of electrical engineering of Japan,” Industry application society, No. 776, 2000.

[12] Y. Jia,“Caffe,” http://caffe.berkeleyvision.org/, 2017/8/19.

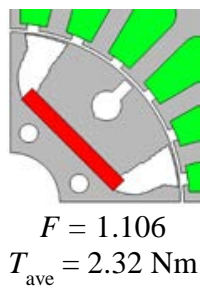

(a) Setting of Table. 5(I)

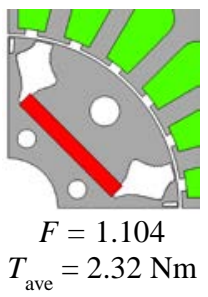

(b) Setting of Table. 5(II)

Fig. 7. Optimization results

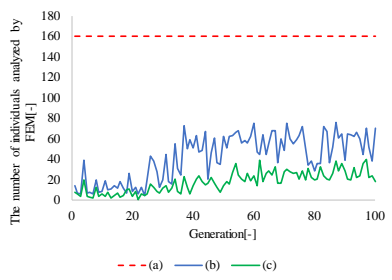

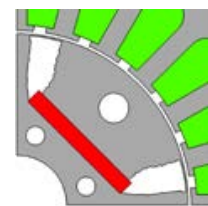

$F=1.101$

$T_{\text {ave }}=2.31 \mathrm{Nm}$

(c) Setting of Table. 5(III) 